\title{
Superior semicircular canal dehiscence syndrome: Diagnostic criteria consensus document of the committee for the classification of vestibular disorders of the Bárány Society
}

\author{
Bryan K. Ward ${ }^{\mathrm{a}, *}$, Raymond van de Berg ${ }^{\mathrm{b}}$, Vincent van Rompaey ${ }^{\mathrm{c}}$, Alexandre Bisdorff ${ }^{\mathrm{d}}$, \\ Timothy E. Hullare, Miriam S. Welgampola ${ }^{\mathrm{f}}$ and John P. Carey ${ }^{\mathrm{a}}$ \\ ${ }^{a}$ Department of Otolaryngology-Head \& Neck Surgery, Johns Hopkins University, Baltimore, USA \\ ${ }^{\mathrm{b}}$ Department of Otorhinolaryngology and Head \& Neck Surgery, Maastricht University Medical Center+, \\ Maastricht, The Netherlands \\ ${ }^{\mathrm{c}}$ Department of Otorhinolaryngology and Head \& Neck Surgery, Antwerp University Hospital, Department of \\ Translational Neurosciences, Faculty of Medicine and Health Sciences, University of Antwerp, Belgium \\ ${ }^{\mathrm{d} C l i n i q u e}$ du Vertige, Centre Hospitalier Emile Mayrisch, Esch-sur-Alzette, Luxembourg \\ ${ }^{\mathrm{e}}$ VA Portland National Center for Rehabilitative Auditory Research and Department of Otolaryngology-Head \\ \& Neck Surgery, Oregon Health \& Science University, Portland, USA \\ ${ }_{\mathrm{f}}^{\mathrm{f}}$ Institute of Clinical Neurosciences, Royal Prince Alfred Hospital, Central Clinical School, University of Sydney, \\ Australia
}

Received 30 June 2020

Accepted 24 November 2020

\begin{abstract}
This paper describes the diagnostic criteria for superior semicircular canal dehiscence syndrome (SCDS) as put forth by the classification committee of the Bárány Society. In addition to the presence of a dehiscence of the superior semicircular canal on high resolution imaging, patients diagnosed with SCDS must also have symptoms and physiological tests that are both consistent with the pathophysiology of a 'third mobile window' syndrome and not better accounted for by another vestibular disease or disorder. The diagnosis of SCDS therefore requires a combination of A) at least one symptom consistent with SCDS and attributable to 'third mobile window' pathophysiology including 1) hyperacusis to bone conducted sound, 2) sound-induced vertigo and/or oscillopsia time-locked to the stimulus, 3) pressure-induced vertigo and/or oscillopsia time-locked to the stimulus, or 4) pulsatile tinnitus; B) at least 1 physiologic test or sign indicating that a 'third mobile window' is transmitting pressure including 1) eye movements in the plane of the affected superior semicircular canal when sound or pressure is applied to the affected ear, 2) low-frequency negative bone conduction thresholds on pure tone audiometry, or 3) enhanced vestibular-evoked myogenic potential (VEMP) responses (low cervical VEMP thresholds or elevated ocular VEMP amplitudes); and C) high resolution computed tomography (CT) scan with multiplanar reconstruction in the plane of the superior semicircular canal consistent with a dehiscence. Thus, patients who meet at least one criterion in each of the three major diagnostic categories (symptoms, physiologic tests, and imaging) are considered to have SCDS.
\end{abstract}

Keywords: Superior canal dehiscence, third mobile window, inner ear, labyrinth

\footnotetext{
${ }^{*}$ Corresponding author: Bryan K. Ward, Department of Otolaryngology-Head \& Neck Surgery, Johns Hopkins University, Baltimore, USA. E-mail: bward15@jhmi.edu.
} 


\section{Introduction}

Patients with a dehiscence in the bone overlying the superior semicircular canal may experience symptoms of pressure- or sound-induced vertigo, hyperacusis to bone conducted sounds, and pulsatile tinnitus. The resulting syndrome, called superior canal dehiscence syndrome (SCDS) includes acute vertigo attacks that are repeatably triggered by particular sounds or pressure changes as well as chronic disequilibrium due to the movement of labyrinthine fluids with intracranial pressure changes that occur with brain pulsations, respiratory variations, or changes in head position.

The initial series of patients with SCDS were diagnosed based on common symptoms, a physical examination finding of eye movements in the plane of the superior semicircular canal when ear canal pressure or loud tones were applied to the ear, and high resolution computed tomography (CT) scan demonstrating a dehiscence in the bone over the superior semicircular canal [38]. The cause of SCDS is unknown. Evidence has favored a congenital arrest of bone development over the top of the superior canal [8]. This may be a necessary but not sufficient condition for a third mobile window because the dura may be insufficiently elastic to transmit pressure until it is thinned or stretched. Indeed, in many cases a second event like head trauma may initiate symptoms, perhaps by shear stress on the overlying dura [37]. Others have argued for an acquired etiology. The finding of osteoclastic activity near the superior semicircular canal has been noted in one temporal bone study [24]. The authors suggested that this indicates that increased bone turnover may lead to reabsorption of previously developed bone and the initiation of a third window.

The additional abnormal opening or 'window' in the labyrinth due to the dehiscence has been called a 'third mobile window', (the oval and round windows are the first two windows). Normally, pressurizing the inner ear via the oval window is relieved at the round window. However, when an additional opening or 'window' into the inner ear occurs at the apex of the superior semicircular canal, the biomechanics of the inner ear are altered. A model of the inner ear including a superior canal dehiscence suggests that sound energy entering the oval window at the stapes is partially shunted away from the cochlea and toward the dehiscence in the superior semicircular canal. At the same time, for bone conducted sounds there might be an abnormal low-impedance pathway for sound/pressure to be transmitted into the inner ear via the dehiscence [46]. This pathophysiology accounts for the low-frequency conductive hearing loss and negative bone conduction thresholds on pure tone audiometry [36], lower thresholds for cervical vestibular-evoked myogenic potentials (VEMPs)[52], higher amplitude responses for ocular VEMPs $[4,16,60]$, and the eye movements that can be observed in the plane of the superior semicircular canal induced by sound or pressure applied to the ear canal or with a Valsalva maneuver [38]. Furthermore, the physiology of a 'third mobile window' links the symptoms that patients experience when both an anatomic dehiscence is present and the above physiologic tests are abnormal [58].

There has been ambiguity in the literature about how patients with SCDS are defined. The prevalence of an anatomic dehiscence in the superior semicircular canal in asymptomatic persons has been estimated from a temporal bone study to be $0.7 \%$ [8], and even higher from radiographic studies [32, 62]. Although the true prevalence of SCDS is unknown, the prevalence of patients with symptoms and signs consistent with SCDS does not appear to be this high. It therefore appears that the presence of an anatomic bony dehiscence is insufficient to generate symptoms in some people. Furthermore, CT imaging tends to overestimate the presence of a dehiscence [15]. One aim of providing a definition of SCDS is to improve the specificity of the selection of patients for research studies. As a result of such research, when clinicians encounter a patient who meets these criteria, the prognosis, prevalence, and likely benefits and risks of interventions can be explained based on a common understanding of the condition. Furthermore, in the care of patients, surgical treatment of SCDS appears to be effective at reducing symptoms [13, 37], improving quality of life [45], and normalizing the abnormal diagnostic tests $[57,60]$. Consensus diagnostic criteria are important to ensure that patients do not receive unnecessary surgery, or conversely, that patients who meet diagnostic criteria are offered appropriate counseling about treatment options.

Since the original description of SCDS a greater understanding of the pathophysiology of 'third window' syndromes has been developed through advancements in higher resolution imaging protocols and increasingly sensitive and specific diagnostic tests for the condition. The diagnostic criteria presented here are based on the classification subcommittee's synthesis of accumulated scientific 
evidence in the approximately twenty years since SCDS was first described.

\section{Methods}

The work presented here is part of an ongoing project to develop an international classification of vestibular disorders (ICVD). The ICVD uses a structured process to develop consensus diagnostic criteria for vestibular symptoms and disorders. The process of establishing criteria is overseen by the Classification Committee of the Bárány Society. For each diagnostic category, an international team of content experts from multiple disciplines is established to propose initial criteria based on the best available scientific evidence. For SCDS, the initial diagnostic criteria were based on the clinical findings in patients who were found to have a dehiscence in the bone overlying the superior semicircular canal and the improvement in both symptoms and signs in patients who had undergone surgical plugging or resurfacing of the superior semicircular canal as therapy. The initial criteria were proposed and circulated to the subcommittee members in February, 2017. Comments were gathered and synthesized with modified criteria presented in Munich to the Classification Committee on March 11, 2017 for tentative approval. The definitions presented here are supported by a process of discussion and refinement as established by the classification committee for the ICVD. The criteria presented below have been carefully considered to account for broad applicability to the international community of otolaryngologists, physical therapists, neurophysiologists, audiologists, neurologists, neurosurgeons and neurotologists who may be seeing patients with this syndrome.

The diagnosis of superior semicircular canal dehiscence syndrome requires all of the following criteria:

1. At least 1 of the following symptoms consistent with the presence of a 'third mobile window' in the inner ear:

1. Bone conduction hyperacusis 1

2. Sound-induced vertigo and/or oscillopsia time-locked to the stimulus ${ }^{2}$

3. Pressure-induced vertigo and/or oscillopsia time-locked to the stimulus ${ }^{3}$

4. Pulsatile tinnitus

2. At least 1 of the following signs or diagnostic tests indicating a 'third mobile window' in the inner ear:
1. Nystagmus characteristic of excitation or inhibition of the affected superior semicircular canal evoked by sound, or by changes in middle ear pressure or intracranial pressure 4

2. Low-frequency negative bone conduction thresholds on pure tone audiometry ${ }^{5}$

3. Enhanced VEMP responses (low cervical VEMP thresholds or high ocular VEMP amplitudes $)^{6}$

3. High resolution temporal bone CT imaging with multiplanar reconstruction demonstrating dehiscence of the superior semicircular canal ${ }^{7}$

4. Not better accounted for by another vestibular disease or disorder.

\section{Notes}

1. Symptoms can include hearing one's voice loudly or distorted in the affected ear (autophony), abnormal perception of one's own internal body sounds like hearing loudly one's eye movements or blinking, borborygmi, crepitus from jaw or neck movements, and footfalls.

2. Sound-induced vertigo and/or oscillopsia (Tullio phenomenon) should be triggered regularly by stimuli that are characteristic to the individual case and ear in question. The vestibular symptoms include dizziness, oscillopsia, or vertigo triggered during exposure to sounds. Provoking sounds tend to be loud, low-frequency sounds. Time-locking to the stimulus implies that the onset and duration of symptoms are linked to the stimulus period.

3. Pressure-induced vertigo and/or oscillopsia can occur from performing Valsalva maneuvers (moderately forceful attempted exhalation against a closed airway, whether by nasal or glottic closure, as when coughing, straining, or sneezing), or when changing the pressure in the ear canal. The vertigo and/or oscillopsia is timelocked with the stimulus and may occur with the application of pressure or its release.

4. Nystagmus is a clinical sign that can be observed using either video-oculography or Frenzel goggles in the clinic. Eye movements should be in the plane of the superior semicircular canal, time-locked with the stimulus. This can be made more obvious by having the patient align their pupil with the plane of the superior semicircular canal, such that a vertical, rather than torsional eye movement is seen (e.g. asking 
the patient to look right when assessing the right ear). An audiometer can be used to administer tones at different frequencies and intensities to each ear. The provocative intensities can be quite loud (e.g. $100 \mathrm{~dB}$ hearing level, HL), so the stimulus should be kept brief. Positive and negative pressure can be delivered to the ear canal with a finger, or by asking the patient to perform nasal Valsalva (pinch the nose closed and blow to generate positive nasopharyngeal pressure) or glottic Valsalva (asking the patient to bear down as if lifting a heavy item) maneuvers.

5. Pure tone threshold audiometry typically shows differences in air and bone conduction thresholds at low to middle frequencies $(250 \mathrm{~Hz}$, $500 \mathrm{~Hz}, 1000 \mathrm{~Hz}$ and sometimes $2000 \mathrm{~Hz}$ ). The most common diagnostic abnormality for SCDS is the presence of one or more negative bone conduction (BC) thresholds, i.e., the individual can perceive the $\mathrm{BC}$ sound stimulus at intensities not normally perceived in the population. However, varying degrees of sensorineural hearing loss may co-exist with SCDS, particularly as patients age. For this reason, the $\mathrm{BC}$ threshold may not be negative but will still be lower than the air conduction (AC) threshold at the same frequency, causing an air-bone gap on the audiogram. This can present a diagnostic challenge because many conditions of the tympanic membrane or middle ear can cause air-bone gaps, a situation commonly referred to as "conductive hearing loss" (CHL). However, middle ear causes of CHL typically produce measurable abnormalities on other tests included in comprehensive audiometry. Immittance testing, which measures the sound energy transferred by the middle ear structures, yields information about the compliance and volume of the middle ear. Air-bone gaps caused by tympanic membrane or middle ear disease typically produce abnormalities of these immittance measures. Conversely, airbone gaps with normal immittance measures could support a diagnosis of SCDS. Likewise, the stapedial reflex test is used to assess the mobility of the stapes, the final entry point into the labyrinth for sound. When the stapes is fixed by otosclerosis or congenital fixation, the stapedial reflex is typically absent. Thus, the presence of air-bone gaps in a patient with normal stapedial reflex testing could also be supportive of a third-mobile window like SCDS. In summary, interpretation of audiometric testing requires a synthesis of the findings of comprehensive audiometry to determine whether or not the presence of low- to mid-frequency air-bone gaps are supportive of the diagnosis of SCDS. Some patients may have auditory symptoms in the absence of vestibular complaints [3], and it is possible to meet the criteria for SCDS with auditory symptoms and findings under criteria 1 and 2.

6. Cervical VEMP (cVEMP) thresholds are decreased on the affected side in patients with SCDS, while ocular VEMP (oVEMP) amplitudes are higher on the affected side in patients with SCDS. VEMP thresholds and amplitudes should be compared to normal ranges established for the particular system/laboratory as there can be a number of variations in the techniques of testing. VEMP results should also be interpreted in light of audiometric findings because VEMPs evoked by air-conducted sound are typically reduced by middle ear causes of CHL. This is due to the loss of sound energy reaching the labyrinth from the oval window. In SCDS one can encounter the presence of robust VEMP responses with an air-bone gap at the stimulus frequency when there is normal immittance and stapedial reflex testing -i.e., when the air-bone gap is not caused by a middle ear cause of CHL. It is again emphasized that all of the findings of VEMP and audiometric testing must be synthesized for a meaningful interpretation.

7. CT scans should preferably have a resolution near $0.2 \mathrm{~mm}$ or better. The images should be reconstructed in the plane of the superior semicircular canal as well as orthogonal to it, to demonstrate the dehiscence.

\section{Comments}

\subsection{Definite versus probable superior semicircular canal dehiscence syndrome}

The committee discussed proposing probable and definite categories of superior semicircular canal dehiscence syndrome. In favor of this approach is the broad range of auditory and vestibular symptoms with which patients may present reported in the literature [39], as well as the possibility for superior semicircular canal dehiscence to be confirmed during surgery. Thus, we considered a separate category 
of "definite SCDS" for cases in which dehiscence was confirmed at the time of surgery. As a diagnostic criterion, however, a surgically confirmed dehiscence is not particularly helpful for patients who have to decide whether they wish to have an intervention. Also, many surgeries are performed via the transmastoid approach, during which surgeons often decide not to confirm the dehiscence e.g. by opening the canal after plugging both ends. We therefore decided against making a distinction between definite and probable SCDS and instead only have one set of criteria for "definite SCDS".

\subsection{The spectrum of presenting symptoms in $S C D S$}

Patients with SCDS present with a variety of symptoms, with some patients having more auditory and others more vestibular complaints. To accommodate the variability of clinical presentation within a single diagnostic category, we proposed that patients are required to have at least one symptom in the category of symptoms that are supported by the pathophysiology of a third mobile window. Bone conduction hyperacusis symptoms are thought to occur from the low impedance pathway caused by the presence of a third mobile window. Patients often report hearing pulsatile tinnitus in the affected ear, likely from transmitted pulsations of the dura through the dehisced semicircular canal. Some authors prefer the more specific term 'pulse-synchronous tinnitus.' This term describes a tinnitus perception completely synchronous in time and phase with the carotid arterial pulse. In contrast, patients with SCDS may hear the pulsation of changes in intracranial pressure or a less pulsatile but more undulating turbulence of blood flow in one of the intracranial venous sinuses, most likely the superior petrosal sinus. In these cases, the frequency of the percept would be the same as the radial pulse, but the phase can be different, and the quality might not even be described as pulsatile. All of these perceptions would be consistent with our use of the commonly accepted clinical term "pulsatile tinnitus" because they are ultimately related to vascular pulsations.

Other symptoms included in the criteria are soundor pressure-induced vertigo that is time-locked to the stimulus. As a result of pressure being transmitted via the oval window toward the dehiscence and across the sensory epithelia of the labyrinth, the onset of symptoms is time-locked with their stimu- lus. Whether the total duration of these symptoms is exactly time-locked with their stimulus, or whether an after-effect might be present, has not yet been thoroughly investigated. Therefore, the committee decided, using expert opinion, that the duration of symptoms is also mainly time locked.

Patients with migraine commonly report hyperacusis to sound (phonophobia) during a migraine with some also experiencing increased vertigo/dizziness with increasing sound exposure. These vestibular symptoms tend not to be time-locked to the stimulus as in SCDS. Furthermore, SCDS is a chronic condition in which symptoms should be triggered regularly whenever the characteristic stimulus is encountered in the affected ear.

There are other symptoms reported by patients with SCDS that are less clearly associated with a 'third mobile window, pathophysiology. These include aural fullness, cognitive effects such as 'brain fog,' chronic disequilibrium, and exacerbation of migraine symptoms, including headaches. Although these symptoms may be related to the dehiscent superior semicircular canal and have been observed to improve after surgery, they tend to improve less frequently [2] and might not be attributable directly to the presence of the dehiscence. Common conditions such as Eustachian tube dysfunction, temporomandibular disorders, and migraine can also cause ear fullness. Other vestibular disorders such as vestibular migraine [30] or persistent postural-perceptual dizziness (PPPD) [51] include symptoms such as 'brain fog', chronic disequilibrium, or headaches and can co-occur with SCDS. These symptoms were therefore not included in the current diagnostic criteria.

\subsection{Unilateral versus bilateral superior semicircular canal dehiscence}

While many patients are symptomatic only on one side, up to $50 \%$ of patients have bilateral anatomic dehiscences [8]. Symptoms and signs of SCDS should localize to the affected ear as much as possible in cases of unilateral SCDS. In patients with bilateral dehiscence, nystagmus elicited by stimuli provided to a single ear should still be characteristic of excitation or inhibition of the superior canal of the stimulated ear. Patients with bilateral anatomic dehiscences tend to have larger air-bone gaps and lower cervical VEMP thresholds in the more symptomatic ear. Additionally, in the Weber tuning fork exam $(512 \mathrm{~Hz}$ tuning fork stimuli) the sound is heard 
more loudly on the symptomatic side [41]. The behavior of each ear should be considered separately on audiometric and VEMP testing and during examination of the patient with ear canal specific sound or pressure stimuli. However, low-frequency bone conduction hyperacusis can prove difficult to localize to one ear during audiometric testing, even with proper masking techniques. In some cases, correction of the dehiscence on the more symptomatic side can lead to apparent resolution of bone conduction hyperacusis on the contralateral side. Therefore, clinicians should be cautious in drawing conclusions about the less symptomatic ear based on auditory testing alone.

\subsection{Near dehiscence}

There have been reports of patients that present with symptoms and signs consistent with SCDS, but who are found at the time of surgery to have thin and in some cases compliant bone overlying the superior semicircular canal rather than a true dehiscence [59]. While evidence supports that even pinpoint dehiscences can alter pressure transmission through the inner ear [42, 44], pinpoint dehiscences are not readily detectable with current imaging technology. Even more controversial is the notion that a physiologic third window can occur in the absence of radiographic abnormalities of the otic capsule [56]. Therefore, the committee decided that additional data are needed on such cases before they can be considered part of ICVD diagnostic criteria for SCDS or other vertigo syndromes.

\subsection{Nystagmus}

The original series of patients with SCDS all had the clinical sign of nystagmus in the plane of the affected superior semicircular canal with loud sound or pressure applied to the affected ear [38]. The observation of nystagmus when these stimuli are provided is explained by the 'third mobile window,' pathophysiology. When loud low-frequency sound or positive pressure is delivered to the affected ear, a pressure gradient occurs between the oval window and the dehiscence, causing an ampullofugal or excitatory input to the affected superior semicircular canal. Increases in intracranial pressure such as during a Valsalva maneuver against a closed glottis causes a pressure gradient from the dehiscence to the oval window, causing an ampullopetal flow or inhibitory input to the affected superior semicircular canal [14].

\subsection{Vestibular-evoked myogenic potentials}

In SCDS, vestibular evoked myogenic potential (VEMP) responses are enhanced. Cervical VEMP (cVEMP) thresholds are decreased in patients with SCDS [60]. The sensitivity and specificity of cVEMP thresholds for SCDS is $>80 \%$ [22,64], but depends on the parameters used. Thus, cutoff values for enhancement of responses must be established for the system used by the particular laboratory. Ocular VEMP (oVEMP) is a newer test that has also been found to be highly sensitive and specific for SCDS. Since it does not rely on a threshold response, the oVEMP is faster to perform, and more easily tolerated. Data suggest the response to $500 \mathrm{~Hz}$ tone bursts may be more sensitive and specific than cVEMP [23, 55, Zuniga et al., 2013). Not all patients have a measurable VEMP, however, and it can be absent as a result of prior middle ear surgery. The VEMP can also be enhanced in other 'third mobile window syndromes,' such as in enlarged vestibular aqueduct syndrome [49]. Similar to acoustic reflexes, VEMPs can be helpful in establishing that a low-frequency conductive hearing loss is from a middle ear source such as otosclerosis, as the VEMP in response to air-conducted sounds should be absent in otosclerosis, and enhanced in cases of SCDS [64].

\subsection{Electrocochleography}

Electrocochleography (ECochG) has been reported by several groups to be abnormal in patients with SCDS, with patients with a dehiscence consistently demonstrating an elevated summating potential (SP) to action potential (AP) ratio [3, 43, 61]. This observation appears to be a marker of a 'third mobile window, as this elevated SP to AP ratio decreases after plugging the dehisced semicircular canal $[1,61]$. ECochG was formerly a popular diagnostic test for Ménière's disease, yet the symptoms of patients presenting with SCDS rarely align with those of patients with Ménière's disease. The committee discussed including an elevated SP to AP ratio in the absence of a low-frequency sensorineural hearing loss as a physiological measure of a third mobile window. Although an elevated SP to AP ratio on ECochG appears to be a consistent finding among patients with superior semicircular canal dehiscence, the other diagnostic tests included in the criteria have more supporting evidence and appear to be more specific for a third mobile window syndrome. Pending additional data such as the sensitivity and specificity of an elevated 
SP to AP ratio on ECochG for SCDS, ECochG could be considered in future versions of the diagnostic criteria.

\subsection{Other physiologic tests}

There are other physiologic measures that have been reported or could be conceived of in the future but were not included in the current diagnostic criteria since they are not commonly available or standardized. Some examples include power reflectance [33], pulsatile oscillations of the eye [53] and pulsatile movements of the tympanic membrane [10,21]. Such diagnostic adjuncts could be considered as supportive evidence for SCDS in centers that have expertise in these recordings and may be promising avenues for further research.

\subsection{CT imaging}

The term "high-resolution" has been applied to a wide variety of CT scanning parameters and continues to change as technology is updated. In a review of temporal bone $\mathrm{CT}$ scans done in a tertiary referral center $9 \%$ of scans had apparent superior semicircular canal dehiscence [62]. Many of these are likely false dehiscences caused by the limits of resolving thin bone, since the prevalence of a dehiscence in a survey of post-mortem temporal bones was only $0.7 \%$ [8]. The same study estimated that absent bone or thin bone (less than $0.1 \mathrm{~mm}$ and likely to look dehiscent on $\mathrm{CT}$ ) occurs in $1.7 \%$ of individuals, meaning that based on CT imaging alone, the population prevalence of a dehiscence would be approximately $2 \%$. A recent study by Berning et al. using higher resolution CT indeed found a population prevalence of superior semicircular canal dehiscence in asymptomatic individuals to be approximately $2 \%$ [5]. Such results can be obtained with CTs that have a spatial resolution near $0.2 \mathrm{~mm}$ or better. This can be accomplished with multidetector CT, the most commonly used technology for temporal bone CT. Collimation of the x-ray beam to $0.5 \mathrm{~mm}$ to $0.63 \mathrm{~mm}$ allows the data to be represented by nearly isotropic voxels so that the images can be reformatted in any plane without distortion. The field of view used to reconstruct the images of the inner ear should be the smallest size possible, so that the labyrinth is displayed to maximal resolution over the fixed size of the image matrix (usually $512 \mathrm{X}$ 512 pixels). Image filters should be set for bone edge detection, since those filters producing less "noisy" images are likely to filter out a thin layer of bone that might remain over the canal. In comparison to multidetector CT, flat-panel CT with a cone beam source of radiation can give even better spatial resolution [54]. After data acquisition by either multidetector or flat-panel CT, images should be reconstructed from a small field of view (FOV) sufficient to encompass the entire inner ear, but small enough so as to not sacrifice spatial resolution. In practice, a FOV that only encompasses the ipsilateral temporal bone gives good resolution. Images should be reconstructed in the plane of the superior semicircular canal as well as orthogonal to it so that any dehiscence can be definitively demonstrated. Ideally, radial reconstructions depicting cross-sections orthogonal to the lumen of the superior semicircular canal give the truest representation of the thickness of the bone over the canal. However, even optimized scans are not without the risks of false positive findings, so the diagnosis of SCDS must never be based on a CT scan alone.

\subsection{Magnetic resonance imaging (MRI)}

Several groups have proposed using MRI as part of the diagnostic workup of SCDS [7, 27]. With newer MRI pulse sequences, improved gradient technology, and higher strength magnetic fields, the resolution of MRI of the inner ear has improved. Current MRI technology, however, has not demonstrated adequate resolution for identifying a dehiscent semicircular canal as compared to high resolution CT imaging [50]. Currently, MRI remains useful for detecting other intracranial anomalies that can cause dizziness, as well as to determine adequacy of prior attempts of plugging the superior semicircular canal in patients with persistent symptoms after surgery [48]. For now, MRI was not included as part of the initial diagnostic workup for patients with symptoms of SCDS.

\subsection{Demographics of superior semicircular canal dehiscence syndrome}

Although the prevalence of an anatomic dehiscence of the superior semicircular canal is $0.7 \%$ [8], the prevalence of superior semicircular canal dehiscence syndrome is unknown. Patients tend to present with symptoms during the fifth and sixth decades of life. SCDS appears to affect both genders similarly, with some studies suggesting higher prevalence in women $[18,41]$ and others higher prevalence in men $[37,22]$. Younger adults and those over age 65 tend to 
present with similar symptoms, as do men and women [40]. Cases of SCDS in children have been identified $[20,29]$. Children tend to report more auditory symptoms and may report hyperacusis or generalized dizziness [29]. Differences in presenting symptoms between children and adults may be attributed to younger children having difficulty explaining their symptoms to adults. Alternatively, changes in the compliance of the dura with age may affect the clinical presentation of SCDS. Additional studies are needed to determine if diagnostic criteria should be adjusted for a pediatric diagnosis of SCDS.

\subsection{Differential diagnosis}

"SCDS-like," presentations can be seen in cases of other causes of a third mobile window.

Patients with these findings may have evidence for third mobile window physiology as evidenced by findings on the diagnostic tests listed in the SCDS criteria, but imaging or context of symptom onset should raise suspicion that the case is atypical.

Secondary SCDS. Cases reported include those caused by meningiomas [12], meningocele [9], or fibrous dysplasia [17]. These cases suggest that any third mobile window into the inner ear may cause similar effects on the labyrinth to what is described above [35]. Although CT imaging is recommended for the diagnosis of SCDS, MRI may be appropriate in the rare cases in which a secondary cause of SCDS is identified.

Dehiscences in other semicircular canals. Cases have been reported of posterior $[19,28]$ or horizontal semicircular canal dehiscence [11], as well as other labyrinthine dehiscences potentially capable of causing symptoms and signs similar to SCDS $[6,25,26$, 34]. The diagnostic criteria proposed here do not include 'third mobile windows' in other semicircular canals. Additional data are needed to determine whether patients with 'third mobile windows,' at other locations present similarly to those with SCDS. It is conceivable that symptoms could vary depending on which direction the sound or pressure energy is diverted through the inner ear.

Perilymphatic fistulae. These may present with sound- or pressure-induced vertigo similar to SCDS but the etiology in these cases is usually evident because symptoms start after stapedotomy, cochlear implantation, barotrauma, or a cholesteatoma involving the labyrinth.

\subsection{Other differential diagnoses}

The differential diagnosis of SCDS can be challenging, since SCDS can present as a co-morbidity of other vestibular disorders, and it can have overlapping symptoms with them [39].

Some patients with Ménière's disease report brief episodes of vertigo triggered by sound and pressure changes. This might result from the membranous labyrinth adhering to the stapes footplate as an effect of advanced hydrops. In these cases, not all criteria for SCDS are fulfilled, and the characteristic symptoms of spontaneous episodes of vertigo, sensorineural hearing loss, and other fluctuating aural symptoms of Ménière's disease are more prominent [31].

In Vestibular migraine and PPPD, patients may complain about sound-induced vertigo and hyperacusis. The duration of the vertigo is longer than it is in SCDS. In vestibular migraine, patients often report that dizziness is gradually worsened by the accumulation of multiple sensory insults, including bright lights, motion of the visual scene, and intense sound, and that the dizziness persists beyond the exposure. In contrast, for sound-induced vertigo in SCDS, vertigo typically is present only during in response to certain loud sounds and only during the exposure to those sounds. The hyperacusis in vestibular migraine and PPPD is an overall hypersensitivity to external sounds, or phonophobia. Both vestibular migraine and PPPD, however, are often present as co-morbidities of SCDS [58].

Patients with patulous Eustachian tube dysfunction can experience auditory symptoms that overlap with those of patients with SCDS, including autophony [47]. While autophony of voice is common in both SCDS and patulous Eustachian tube dysfunction, autophony for nasal breathing is less common in SCDS [63] but frequently found in patients with patulous Eustachian tube dysfunction.

\section{References}

[1] M.E. Adams, P.R. Kileny, S.A. Telian, H.K. El-Kashlan, K.D. Heidenreich, G.R. Mannarelli and H.A. Arts, Electrocochleography as a diagnostic and intraoperative adjunct in superior semicircular canal dehiscence syndrome, Otology \& Neurotology 32(9) (2011), 1506-1512.

[2] M.S. Alkhafaji, S. Varma, S.E. Pross, J.D. Sharon, J.C. Nellis, C.C. Della Santina, L.B. Minor and J.P. Carey, Long-Term Patient-Reported Outcomes After Surgery for Superior Canal Dehiscence Syndrome, Otology \& Neurotology 38(9) (2017), 1319-1326. 
[3] H.A. Arts, M.E. Adams, S.A. Telian, H. El-Kashlan and P.R. Kileny, Reversible electrocochleographic abnormalities in superior canal dehiscence, Otology \& Neurotology 30(1) (2009), 79-86.

[4] S.T. Aw, M.J. Todd, G.E. Aw, J.S. Magnussen, I.S. Curthoys and G.M. Halmagyi, Click-evoked vestibulo-ocular reflex Stimulus-response properties in superior canal dehiscence, Neurology 66(7) (2006), 1079-1087.

[5] A.W. Berning, K. Arani and B.F. Branstetter, Prevalence of Superior Semicircular Canal Dehiscence on HighResolution CT Imaging in Patients without Vestibular or Auditory Abnormalities, AJNR 40(4) (2019), 709-712.

[6] D.M. Blake, S. Tomovic, A. Vazquez, H.-J. Lee and R.W. Jyung, Cochlear-facial dehiscence: a newly described entity, The Laryngoscope 124(1) (2014), 283-289.

[7] P. Browaeys, T.L. Larson, M.L. Wong and U. Patel, Can MRI replace $\mathrm{CT}$ in evaluating semicircular canal dehiscence? AJNR 34(7) (2013), 1421-1427.

[8] J.P. Carey, L.B. Minor and G.T. Nager, Dehiscence or thinning of bone overlying the superior semicircular canal in a temporal bone survey, Archives of Otolaryngology-Head \& Neck Surgery 126(2) (2000), 137-147.

[9] A. Castellucci, C. Brandolini, V. Del Vecchio, D. Giordano, C. Pernice, G. Bianchin, V. Maiolo and G.G. Ferri, Temporal Bone Meningocele Associated With Superior Canal Dehiscence, Otology \& Neurotology 39(6) (2018), e506-e508.

[10] A. Castellucci, C. Brandolini, G. Piras, I.J. Fernandez, D. Giordano, C. Pernice, G.C. Modugno, A. Pirodda and G.G. Ferri, Superior canal dehiscence with tegmen defect revealed by otoscopy: Video clip demonstration of pulsatile tympanic membrane, Auris, Nasus, Larynx 45(1) (2018), $165-169$.

[11] T. Cawthorne, Otosclerosis, The Journal of Laryngology and Otology 69(7) (1955), 437-456.

[12] B.T. Crane, J.P. Carey, S. McMenomey and L.B. Minor, Meningioma causing superior canal dehiscence syndrome, Otology \& Neurotology 31(6) (2010), 1009-1010.

[13] B.T. Crane, F.R. Lin, L.B. Minor and J.P. Carey, Improvement in autophony symptoms after superior canal dehiscence repair, Otology \& Neurotology 31(1) (2010), 140-146.

[14] P.D. Cremer, L.B. Minor, J.P. Carey and C.C. Della Santina, Eye movements in patients with superior canal dehiscence syndrome align with the abnormal canal, Neurology 55(12) (2000), 1833-1841.

[15] H.D. Curtin, Superior semicircular canal dehiscence syndrome and multi-detector row CT, Radiology 226(2) (2003), 312-314.

[16] T.D. Fife, J.G. Colebatch, K.A. Kerber, K. Brantberg M. Strupp, H. Lee, M.F. Walker, E. Ashman, J. Fletcher, B. Callaghan and D.S. Gloss, Practice guideline: Cervical and ocular vestibular evoked myogenic potential testing: Report of the Guideline Development, Dissemination, and Implementation Subcommittee of the American Academy of Neurology, Neurology 89(22) (2017), 2288-2296.

[17] J.C. Goddard, J.L. Go and R.A. Friedman, Fibrous Dysplasia Causing Superior Canal Dehiscence, Otology \& Neurotology 34(1) (2013), e1-e2.

[18] J.C. Goddard and E.P. Wilkinson, Outcomes following Semicircular Canal Plugging, Otolaryngology-Head and Neck Surgery 151(3) (2014), 478-483.

[19] Q. Gopen, G. Zhou, D. Poe, M. Kenna and D. Jones, Posterior semicircular canal dehiscence: first reported case series, Otology \& Neurotology 31(2) (2010), 339-344.
[20] S.C.A. Hegemann and J.P. Carey, Is Superior Canal Dehiscence Congenital or Acquired? A Case Report and Review of the Literature, Otolaryngologic Clinics of North America 44(2) (2011), 377-382.

[21] T.E. Hullar, Vascular pulsations on impedance audiometry as a sign of a third-mobile window lesion, Otology \& Neurotology 31(4) (2010), 565-566.

[22] J.B. Hunter, N.S. Patel, B.P. O'Connell, M.L. Carlson, N.T. Shepard, D.L. McCaslin and G.B. Wanna, Cervical and Ocular VEMP Testing in Diagnosing Superior Semicircular Canal Dehiscence, Otolaryngology-Head and Neck Surgery 156(5) (2017), 917-923.

[23] K.L. Janky, K.D. Nguyen, M. Welgampola, M.G. Zuniga and J.P. Carey, Air-conducted oVEMPs provide the best separation between intact and superior canal dehiscent labyrinths, Otology \& Neurotology 34(1) (2013), 127-134.

[24] T. Kamakura and J.B. Nadol, Evidence of Osteoclastic Activity in the Human Temporal Bone, Audiology \& NeuroOtology 22(4-5) (2017), 218-225.

[25] M. Karlberg, M. Annertz and M. Magnusson, Mondini-like malformation mimicking otosclerosis and superior semicircular canal dehiscence, The Journal of Laryngology and Otology 120(5) (2006), 419-422.

[26] H.H.S. Kim and D.F. Wilson, A third mobile window at the cochlear apex, Otolaryngology-Head and Neck Surgery 135(6) (2006), 965-966.

[27] G.A. Krombach, E. Di Martino, S. Martiny, A. Prescher, P. Haage, A. Buecker and R.W. Günther, Dehiscence of the superior and/or posterior semicircular canal: delineation on T2-weighted axial three-dimensional turbo spin-echo images, maximum intensity projections and volume-rendered images, European Archives of Oto-RhinoLaryngology 263(2) (2006), 111-117.

[28] G.A. Krombach, E. DiMartino, T. Schmitz-Rode, A. Prescher, P. Haage, S. Kinzel and R.W. Günther, Posterior semicircular canal dehiscence: a morphologic cause of vertigo similar to superior semicircular canal dehiscence, European Radiology 13(6) (2003), 1444-1450.

[29] C. Lagman, V. Ong, L.K. Chung, L. Elhajjmoussa, C. Fong, A.C. Wang, Q. Gopen, and I. Yang, Pediatric superior semicircular canal dehiscence: illustrative case and systematic review, Journal of Neurosurgery: Pediatrics 20(2) (2017), 196-203.

[30] T. Lempert, J. Olesen, J. Furman, J. Waterston, B. Seemungal, J.P. Carey, A. Bisdorff, M. Versino, S. Evers and D. Newman-Toker, Vestibular migraine: diagnostic criteria, Journal of Vestibular Research: Equilibrium \& Orientation 22(4) (2012), 167-172.

[31] J.A. Lopez-Escamez, J.P. Carey, W.-H. Chung, J.A. Goebel, M. Magnusson, M. Mandalà, D.E. Newman-Toker, M. Strupp, M. Suzuki, F. Trabalzini, A. Bisdorff, Diagnostic criteria for Menière's disease, Journal of Vestibular Research: Equilibrium \& Orientation 25(1) (2015), 1-7.

[32] Y. Masaki, The prevalence of superior canal dehiscence syndrome as assessed by temporal bone computed tomography imaging, Acta Oto-Laryngologica 131(3) (2011), 258-262.

[33] G.R. Merchant, C. Röösli, M.E.F. Niesten, M.A. Hamade, D.J. Lee, M.L. McKinnon, C.H. Ulku, J.J. Rosowski, S.N. Merchant and H.H. Nakajima, Power reflectance as a screening tool for the diagnosis of superior semicircular canal dehiscence, Otology \& Neurotology 36(1) (2015), 172-177.

[34] S.N. Merchant, H.H. Nakajima, C. Halpin, J.B. Nadol, Jr, D.J. Lee, W.P. Innis, H. Curtin and J.J. Rosowski, Clinical investigation and mechanism of air-bone gaps in large 
vestibular aqueduct syndrome, The Annals of Otology, Rhinology, and Laryngology 116(7) (2007), 532-541.

[35] S.N. Merchant and J.J. Rosowski, Conductive hearing loss caused by third-window lesions of the inner ear, Otology \& Neurotology 29(3) (2008), 282-289.

[36] A.A. Mikulec, M.J. McKenna, M.J. Ramsey, J.J. Rosowski, B.S. Herrmann, S.D. Rauch, H.D. Curtin and S.N. Merchant, Superior semicircular canal dehiscence presenting as conductive hearing loss without vertigo, Otology \& Neurotology 25(2) (2004), 121-129.

[37] L.B. Minor, Clinical manifestations of superior semicircular canal dehiscence, The Laryngoscope 115(10) (2005), 1717-1727.

[38] L.B. Minor, D. Solomon, J.S. Zinreich and D.S. Zee, Sound- and/or pressure-induced vertigo due to bone dehiscence of the superior semicircular canal, Archives of Otolaryngology-Head \& Neck Surgery 124(3) (1998), 249-258.

[39] L. Naert, R. Van de Berg, P. Van de Heyning, A. Bisdorff, J.D. Sharon, B.K. Ward and V. Van Rompaey, Aggregating the symptoms of superior semicircular canal dehiscence syndrome, The Laryngoscope 128(8) (2017), 1932-1938.

[40] T. Nguyen, J.P. Sheppard, C. Duong, K. Ding, D. Dejam, Y. Alkhalid, P. Romiyo, D. Azzam, G. Prashant, Q. Gopen and I. Yang, Age and gender considerations on the symptomology in patients with superior semicircular canal dehiscence: A systematic review and case illustration, Journal of Clinical Neuroscience 65 (2019), 112-120.

[41] M.E.F. Niesten, M.J. McKenna, B.S. Herrmann, W. Grolman and D.J. Lee, Utility of cVEMPs in bilateral superior canal dehiscence syndrome, The Laryngoscope 123(1) (2013), 226-232.

[42] M.E.F. Niesten, C. Stieger, D.J. Lee, J.P. Merchant, W. Grolman, J.J. Rosowski and H.H. Nakajima, Assessment of the effects of superior canal dehiscence location and size on intracochlear sound pressures, Audiology \& Neuro-Otology 20(1) (2015), 62-71.

[43] J.H. Park, S.Y. Lee, J.-J. Song, B.Y. Choi and J.-W. Koo, Electrocochleographic findings in superior canal dehiscence syndrome, Hearing Research 323 (2015), 61-67.

[44] D.V. Pisano, M.E.F. Niesten, S.N. Merchant and H.H. Nakajima, The effect of superior semicircular canal dehiscence on intracochlear sound pressures, Audiology \& Neuro-Otology 17(5) (2012), 338-348.

[45] A.K. Remenschneider, M. Owoc, E.D. Kozin, M.J. McKenna, D.J. Lee and D.H. Jung, Health Utility Improves After Surgery for Superior Canal Dehiscence Syndrome, Otology \& Neurotology 36(10) (2015), 1695-1701.

[46] J.J. Rosowski, J.E. Songer, H.H. Nakajima, K.M. Brinsko and S.N. Merchant, Clinical, experimental, and theoretical investigations of the effect of superior semicircular canal dehiscence on hearing mechanisms, Otology \& Neurotology 25(3) (2004), 323-332.

[47] A.G.M. Schilder, M.F. Bhutta, C.C. Butler, C. Holy, L.H. Levine, K.J. Kvaerner, G. Norman, R.J. Pennings, D. Poe, J.T. Silvola, H. Sudhoff and V.J. Lund, Eustachian tube dysfunction: consensus statement on definition, types, clinical presentation and diagnosis, Clinical Otolaryngology 40(5) (2015), 407-411.

[48] J.D. Sharon, S.E. Pross, B.K. Ward and J.P. Carey, Revision Surgery for Superior Canal Dehiscence Syndrome, Otology \& Neurotology 37(8) (2016), 1096-1103.

[49] K. Sheykholeslami, S. Schmerber, M. Habiby Kermany and K. Kaga, Vestibular-evoked myogenic potentials in three patients with large vestibular aqueduct, Hearing Research 190(1-2) (2004), 161-168.

[50] S.A. Spear, N.M. Jackson, R. Mehta, C.E. Morel, L.S. Miller, D. Anderson and M.A. Arriaga, Is MRI Equal to CT in the Evaluation of Thin and Dehiscent Superior Semicircular Canals? Otology \& Neurotology 37(2) (2016), 167-170.

[51] J.P. Staab, A. Eckhardt-Henn, A. Horii, R. Jacob, M. Strupp, T. Brandt and A. Bronstein, Diagnostic criteria for persistent postural-perceptual dizziness (PPPD): Consensus document of the committee for the classification of Vestibular Disorders of the Bárány Society, Journal of Vestibular Research: Equilibrium \& Orientation 27(4) (2017), 191-208.

[52] S.O. Streubel, P.D. Cremer, J.P. Carey, N. Weg and L.B. Minor, Vestibular-evoked myogenic potentials in the diagnosis of superior canal dehiscence syndrome, Acta OtoLaryngologica, Supplementum 545 (2001), 41-49.

[53] C. Tilikete, P. Krolak-Salmon, E. Truy and A. Vighetto, Pulse-synchronous eye oscillations revealing bone superior canal dehiscence, Annals of Neurology 56(4) (2004), 556-560.

[54] A. Tunkel, J.P. Carey and M. Pearl, Flat Panel Computed Tomography in the Diagnosis of Superior Semicircular Canal Dehiscence Syndrome, Otology \& Neurotology 40(2) (2019), 213-217.

[55] L. Verrecchia, K. Brantberg, Z. Tawfique and D. Maoli, D. Diagnostic Accuracy of Ocular Vestibular Evoked Myogenic Potentials for Superior Canal Dehiscence Syndrome in a Large Cohort of Dizzy Patients, Ear and Hearing 40(2) (2019), 287-294.

[56] P.A. Wackym, S.J. Wood, D.A. Siker and D.M. Carter, Otic capsule dehiscence syndrome: Superior semicircular canal dehiscence syndrome with no radiographically visible dehiscence, Ear, Nose, \& Throat Journal 94(8) (2015), E8-E24.

[57] B.K. Ward, Y. Agrawal, E. Nguyen, C.C. Della Santina, C.J. Limb, H.W. Francis, L.B. Minor and J.P. Carey, Hearing outcomes after surgical plugging of the superior semicircular canal by a middle cranial fossa approach, Otology \& Neurotology 33(8) (2012), 1386-1391.

[58] B.K. Ward, J.P. Carey and L.B. Minor, Superior Canal Dehiscence Syndrome: Lessons from the First 20 Years, Frontiers in Neurology 8 (2017), 177.

[59] B.K. Ward, A. Wenzel, E.K. Ritzl, S. Gutierrez-Hernandez, C.C. Della Santina, L.B. Minor and J.P. Carey, Neardehiscence: clinical findings in patients with thin bone over the superior semicircular canal, Otology \& Neurotology 34(8) (2013), 1421-1428.

[60] M.S. Welgampola, O.A. Myrie, L.B. Minor and J.P. Carey, Vestibular-evoked myogenic potential thresholds normalize on plugging superior canal dehiscence, Neurology 70(6) (2008), 464-472.

[61] A. Wenzel, B.K. Ward, E.K. Ritzl, S. Gutierrez-Hernandez, C.C. Della Santina, L.B. Minor and J.P. Carey, Intraoperative neuromonitoring for superior semicircular canal dehiscence and hearing outcomes, Otology \& Neurotology 36(1) (2015), 139-145.

[62] R.A. Williamson, J.T. Vrabec, N.J. Coker and M. Sandlin, Coronal computed tomography prevalence of superior semicircular canal dehiscence, Otolaryngology-Head and Neck Surgery 129(5) (2003), 481-489.

[63] G. Zhou, Q. Gopen and D.S. Poe, Clinical and diagnostic characterization of canal dehiscence syndrome: a great otologic mimicker, Otology \& Neurotology 28(7) (2007), 920-926. 
[64] G. Zhou, D. Poe and Q. Gopen, Clinical use of vestibular evoked myogenic potentials in the evaluation of patients with air-bone gaps, Otology \& Neurotology 33(8) (2012), 1368-1374.
[65] M.G. Zuniga, K.L. Janky, K.D. Nguyen, M.S. Welgampola and J.P. Carey. Ocular versus cervical VEMPs in the diagnosis of superior semicircular canal dehiscence syndrome, Otology \& Neurotology 34(1) (2013), 121-126. 